\title{
Detection of Vulnerable Road Users in Smart Cities
}

\author{
Francisco Guayante, Arnoldo Díaz-Ramírez \\ Department of Computer Systems \\ Instituto Tecnológico de Mexicali \\ Av. Tecnológico s/n Col. Elías Calles \\ Mexicali, B.C., México 21376 \\ \{fguayante, adiaz\}@itmexicali.edu.mx
}

\author{
Pedro Mejía-Alvarez \\ Department of Computer Sciences \\ CINVESTAV-IPN \\ Av. Instituto Politécnico Nacional 2508, Col. Zacatenco \\ México, D.F. 07360 \\ pmalvarez@delta.cs.cinvestav.mx
}

\begin{abstract}
Starting from 2008, more than half of the world's population now lives in urban areas, and this number is expected to grow for the next decades. To the extent that the population of a city grows, new problems arise, which include scarcity of resources, pollution, and traffic congestion. One of the most important problems of big cities are road traffic injuries, which is the eighth leading cause of death globally, and the main cause of death for young people, mainly in middle and low income countries. Vulnerable road users (VRUs) are among the users at higher risks of traffic accidents. In order to cope with the problems of the growing urban communities, the concept of smart cities has emerged. A smart city is based on the use of smart computing technologies, such as Intelligent Transportation Systems and Vehicular Ad hoc Networks. In this paper, we propose a model to be used in smart cities, to detect if a VRU intends to cross a road in a risky zone, and to issue alerts to the vehicles nearby. The proposed model is cost effective, and is able to detect a VRU at risk in a short period of time. The evaluation of the proposed model shows that it performs correctly.
\end{abstract}

Index Terms-Smart Cities, VRUs detection, VANETs

\section{INTRODUCTION}

Nowadays, more than half of the world's population lives in urban areas, and the migration from rural zones to cities is expected to continue for the next decades [1]. However, bigger cities generate new kinds of problems, such as scarcity of resources, pollution, health, traffic congestion and inadequate infrastructure, to mention a few.

One of the most important problems of big cities are road traffic injuries, which is the eighth leading cause of death globally, and the main cause of death for young people. Every year, more than 1.2 million people die on the world's roads, and 50 million people are injured. Middle-income countries have $52 \%$ of the world's vehicles, and $80 \%$ of the world's road traffic deaths [2]. Also, there are different road user groups, with differences in fatality rates among them. The users at greatest risk are the vulnerable road users (VRU's), such as pedestrian and cyclists. In middle and low income countries, the rate of VRUs deaths is greater, in part because less educated people disobey traffic signals and cross streets through dangerous zones.

To face the problems generated by the rapid urban population growth, recently we have seen an interest in the use of the information and communication technology (ICT) to make a city smarter. A smart city is defined by Washburn et al. as "the use of smart computing technologies to make the critical infrastructure components and services of a city -which include city administration, education, healthcare, public safety, real estate, transportation, and utilities- more intelligent, interconnected, and efficient" [13]. Smart computing is referred as "a new generation of integrated hardware, software, and network technologies that provide IT systems with real-time awareness of the real world and advanced analytics to help people make more intelligent decisions about alternatives and actions that will optimize business processes and business balance sheet results" [13].

In this paper, we propose a model to detect if a VRU intends to cross a road in a risky zone, and to issue alerts directed to the vehicles nearby. The proposed model was developed in the context of smart cities. It is based on the use of two emerging technologies: the Intelligent Transportation Systems (ITS) paradigm [8], which consists in the use of information and communication-based solutions to improve the interaction between vehicles and road users; and the Vehicular Ad hoc Networks (VANETs) technology [3]. VANETs comprise vehicle-to-vehicle (V2V) and vehicle-to-infrastructure (V2I) communication based on wireless local area network technologies.

This paper is organized as follows: Section I presents an overview of the related work. Section II introduces the proposed system. In Section III, the results of its evaluation are shown. Finally, the conclusions and future work are discussed in Section V.

\section{RELATED WORK}

In the past few years, some proposals have been published to protect pedestrians using systems based on information technology solutions. These proposals may fall into one of the three categories proposed by Carsten in [4]: in-vehicle systems, carried-by-the-pedestrian systems, and indirect systems.

\section{A. In-vehicle systems}

In-vehicle systems are constituted of sets of sensors or cameras, and processing units mounted within the vehicles, for 
the purpose of detecting VRUs. These systems alert drivers when risky situations are detected, and suggest or perform evasive actions. One drawback of these kind of systems is the short range of detection of the sensors within the vehicle, as well as the signal's distortion generated at high speeds. Also, some measurements may be incomplete due to the presence of other vehicles, which may interfere with the detection of the VRUs.

In [6], Fuerstenberg et al. introduced a pedestrian detection and classification system. Their proposal uses laser scanner sensors, which allows a 180 degrees horizontal field of view. Based on the images taken by the laser scanners, several pedestrian recognition algorithms are able to process signals from objects moving at high speeds, even over long distances. The pedestrians movements may be predicted to determine whether or not they are at risk.

In [7], Garate et al. proposed a support system for drivers using sensor fusion techniques, to process the data collected by a short-range radar, and the monochrome images captured by a camera. The radar system is placed in the center of the front of the vehicle, and has a single beam with a range of 40 meters, and a horizontal view of 50 degrees. It operates at a frequency of $100 \mathrm{~Hz}$, and it is capable to detect up to 32 objects. Using the information obtained by the radar (distance and angle position of the vehicle), and the vehicle speed, it is possible to determine if the detected object is in danger of a collision.

\section{B. Carried-by-the-pedestrian systems}

To detect pedestrians and prevent them from any roadrelated risk, some components of these kind of systems must be carried by the VRUs. The proliferation of smartphones and similar mobile devices allows the implementation of carriedby-the-pedestrian systems. However, the system may not work if the pedestrian does not carry the device.

In [5], Fardi et al., proposed a cooperative sensing system composed of an infrared camera placed inside the vehicle, and Wireless Personal Area (WPAN) modules, to determine the relative position of the VRU. The vehicle and the VRUs are equipped with these modules, respectively. The data collected by the sensors are processed using data fusion methods, with the objective of achieving a reliable real-time detection of VRUs around the vehicle. As a consequence of the detection of a VRU at risk, a warning message is sent, directed to both the driver and the VRUs.

A Collaborative Context Predictor was introduced by Voigtmann et al. in [12]. They proposed a proactive approach to detect pedestrians who are dangerously close to the street, and try to predict the pedestrian's next step using her/his context information (movement and orientation). The pedestrians must carry a smartphone in their left trouser pocket, to gather movement data. The objective of the proposed system is to detect a risk situation in a very short period of time, to avoid a collision.

\section{Indirect systems}

These systems are aimed to improve the interaction between the VRUs and the vehicles, through the use of detection systems connected to the road infrastructure.

Meissner et al. in [10] proposed a method to prevent pedestrian accidents at intersections. To recognize and track moving objects, a network of laser scanners is deployed several meters above the ground, allowing an aerial view of the area of interest. The network is able to generate a three-dimensional profile of the scene, regardless of the light conditions. The method is able to distinguish dynamic objects (pedestrians) from static objects. The pedestrians are identified by means of density and dimensions of the points detected by the sensors. Once the system has detected a pedestrian, the information regarding his movement and position is transmitted to the vehicle using V2I communication. The sensors and the algorithms used allow the detection and tracking of multiple pedestrians.

In [9], Hugues et al. proposed a detection system, where microwave detectors are placed in traffic signals to identify pedestrians at the time they approach to a crossing. The information generated by the sensors was used to make time alterations in traffic signals for vehicles, in order to improve the safety of pedestrians. The results showed that this is a viable method because the microwave sensor can detect a moving object in a way that the system is capable to take fast decisions.

\section{PRoposed SySTEM}

As mentioned previously, our proposal is based on the Intelligent Transportation Systems (ITS) paradigm, and on the Vehicular Ad hoc Networks (VANETs) technology. VANETs communication is based on the IEEE 802.11p protocol, which is an amendment to the IEEE 802.11 standard to add wireless access in vehicular environments (WAVE). In a VANET, vehicles communicate with other vehicles using the vehicleto-vehicle $(\mathrm{V} 2 \mathrm{~V})$ model. Also, vehicles communicate with fixed equipment next to the road, referred to as road side unit (RSU), using the vehicle-to-infrastructure model (V2I). The main system components of the V2I model are the application unit (AU), the on board unit (OBU), and the roadside unit (RSU). Commonly the RSU hosts an application that provides some services that are used by the AU, which is a device equipped within the vehicle. The AU uses the communication capabilities of the OBU, which is a WAVE device mounted on the vehicle, aimed for exchanging information with RSUs or other OBUs [3].

According to Sherborne [11], a VRU detection system based on the indirect system model, should consider different scenarios where the vehicle and the pedestrian interact. In particular, a pedestrian detection system at crossings points should be able to:

- Identify pedestrians approaching to the crossing point.

- Identify pedestrians waiting to cross the road.

- Obtain a measure of how many pedestrians intend to cross the road.

- Identify the situations when pedestrians no longer wish to cross the road.

- Identify the situations when all pedestrians have crossed the road.

Based on these observations, we propose a VRUs detection and monitoring system for smart cities, to be used at risky 


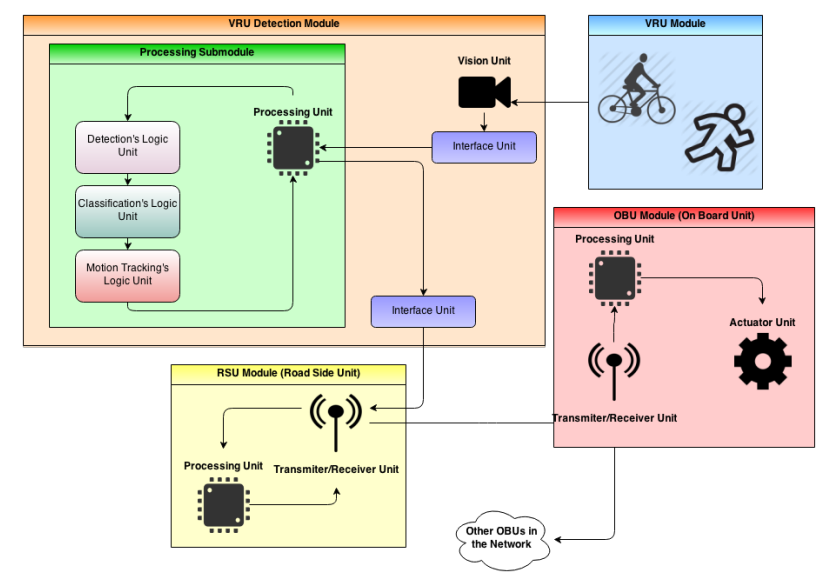

Figure 1. System architecture

or prohibited crossing areas. This system has been developed using the following design guidelines:

- It is based on the indirect system model. This ensures that the VRU is detected without the need to carry any device with him. In contrast to the in-vehicle system model, indirect systems offer a better perspective of the situation, and are able to alert vehicles long before they reach the risk zone.

- It is based on the V2I architecture. The proposed system sends alerts to vehicles when a VRU at risk is detected, using the VANET's technology.

- The system is affordable. Many of the already proposed systems are attractive and efficient. However, they are based on the use of very expensive sensors. One key design concern of the proposed system is cost.

- The system has a low computational complexity. Thus, it is able to detect, track and determine the intentions of pedestrians in a short period of time.

The architecture of the proposed system is shown in Fig. 1. As it can be observed, it is comprised of four modules, which are described next.

\section{A. VRU detection module}

This module is responsible of performing the VRUs detection functions, as well as the classification functions. The module consists of a vision unit, two communication interface units, and a processing submodule.

Vision unit: The vision unit must determine if one or more VRUs are in the detection zone. The sensors used should allow the system, by means of the acquired data, to determine if an object is in motion, if it is a VRU or not, and the motion's direction of the detected VRU.

As discussed previously, many of the proposals that have been published to date use complex techniques (e.g. image processing), use expensive sensors, or may require drastic changes to the road infrastructure. In contrast, the proposed model is able to alert vehicles when a VRU at risk is detected, which requires the use of low complexity algorithms. Additionally, the system uses low cost and highly available sensors. Since it is based on the V2I communication model, it uses the VANET's infrastructure.

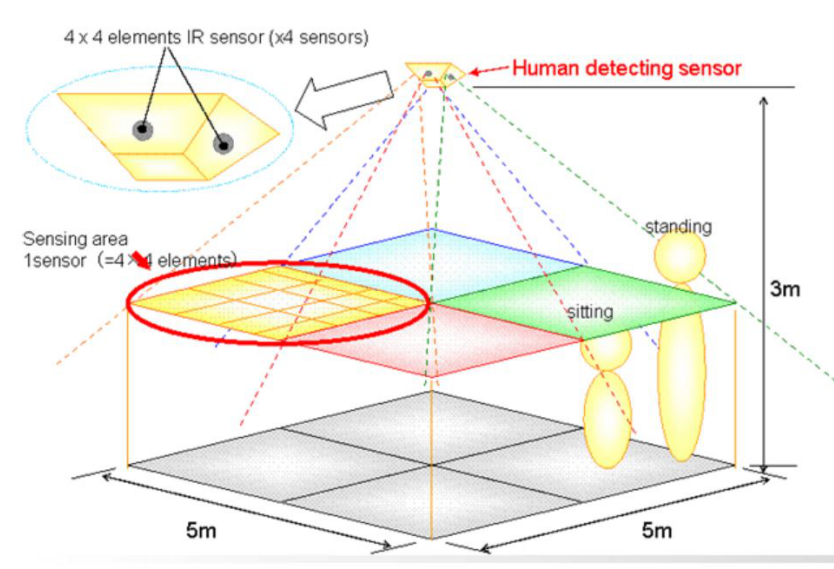

Figure 2. Detection system using four non-contact temperature sensors

In order to select the most suitable sensors to successfully detect VRUs, we evaluated different types of sensors. Some of the them were the Passive InfraRed (PIR) sensors, which measure the infrared light emanating from objects. They are cheap sensors that detect the presence of heat from an object or body nearby. They are also capable of detecting the movement of people when a temperature change occurs. Motion detectors usually use PIR sensors. However, the use of PIR sensors does not allow the detection of multiple pedestrians in the sensed area. Additionally, they are not able to detect a pedestrian if he is not moving, and if the body temperature is similar to the ambient temperature, the pedestrian would not be detected.

In contrast, non-contact temperature sensors have many of the advantages of the PIR sensors, and overcome many of their disadvantages. They are constituted by a set of temperature sensors distributed in a matrix. Each temperature sensor has an angle of view, and it is capable of measuring the heat radiation from the surface of an object. The advantage of these sensors, in contrast with other infrared sensors, is that the latter are not able to detect static objects because they only detect drastic $r$ temperature changes. The non-contact infrared temperature sensors can detect an object whether or not an object is moving.

These devices are constituted by multiple sensors, in a way that the output resembles a low resolution thermal image. This allows not only to detect motion but also to determine in which direction the detected objects are moving, and to calculate the distance between the object and the sensor. Additionally, the use of several sensors may increase the detection area, as shown in Fig. 2.

In the proposed system, we assume the use of one or more non-contact temperature sensors.

Processing submodule: It consists of both physical and logical components that are responsible of processing the data collected by the vision unit. It comprises a processor unit, and three algorithms: a detection algorithm, a classification algorithm and a tracking algorithm.

The processor unit (a microprocessor or computer), is where the algorithms are executed to process the data gathered by the sensor(s). The detection algorithm determines if there is a moving object in the monitored area. The classification 
algorithm uses the information provided by the detection algorithm as input, compares the data with a temperature threshold, and determines whether or not the moving object is a pedestrian. If a VRU is detected, the tracking algorithm follows his movement in order to determine if he is directed towards a risk zone (the street), if he is moving away from the danger zone, or if he is going out of the detection area.

Interface units: These units are composed of hardware and software resources, that allow the connection between the system's components. For instance, an interface unit connects the vision unit and the drive processor submodule. Also, another interface unit connects the processor submodule to the road side unit module.

\section{B. Road Side Unit (RSU) Module}

The RSU module is part of the road infrastructure. It is able to process the data gathered by the sensor(s), and to send messages directed to the vehicles. Once the detection module has generated a warning, it is sent to the RSU module, which in turn will send the alert message to the vehicles nearby. The vehicles that receive a warning message will re-transmit it to other vehicles using the $\mathrm{V} 2 \mathrm{~V}$ model. These messages are received by the vehicle's OBUs.

\section{On Board Unit $(\mathrm{OBU})$ Module}

It is the unit installed within the vehicle, which is responsible for receiving information from the RSUs, or from other OBUs. The OBU module is composed of a transmitter/receiver unit, a submodule processor, and several submodules or actuator units. In this case, when the system detects a VRU trying to cross the street, the OBU will receive the alert message, and will send it to the AU, which will inform the driver about the risk situation.

\section{Proposed algorithms}

As already mentioned, within the processor submodule there are three logical units that allow the system to perform its functions properly. This strongly depends on the proposed algorithms within the submodule. Regarding the algorithms, it is important to point out that they were designed and developed assuming the use of an array of non-contact temperature sensors.

Master algorithm: It is in charge of the decision taking process, once the data have been collected by the vision unit. In addition, it is responsible for storing the data measured by the sensor array.

Detection algorithm: This algorithm compares the current measurements captured by the system, with the prior cycle measurements for each sensor array element. If the difference between the current measurement and the previous one is located within a defined detection threshold, the algorithm determines that there is a moving object within the detection zone. In that case, the classification algorithm is invoked.

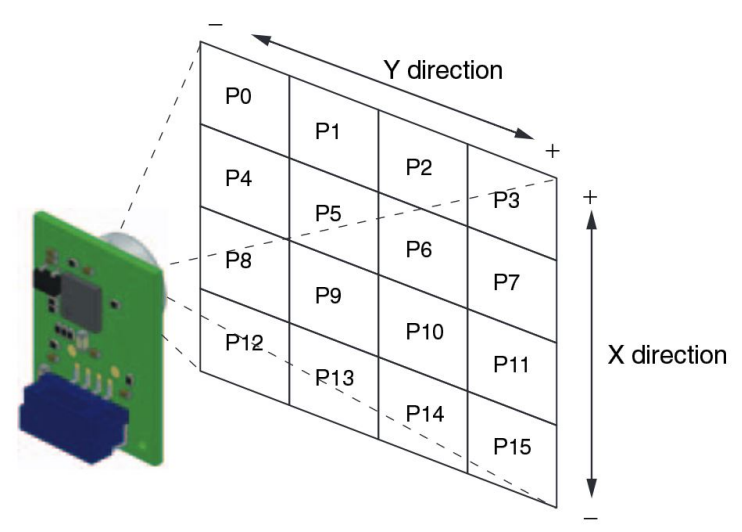

Figure 3. Distribution of the OMRAN D6T-44L device

Classification algorithm: If the detection algorithm determines that there is a moving object within the detection zone, then the classification algorithm checks if the temperature of the moving object is within the temperature range characteristic of a human being. If so, the information is sent to the next process.

Tracking algorithm: Once the system has detected the presence of a VRU, the tracking algorithm checks element by element of the array, compares previous measurements with current ones, to determine in which direction the VRU moves. An example of the detection zone is shown in Fig. 5.

\section{EVALUATION}

In order to evaluate the proposed model, we built a prototype, which is discussed next.

\section{A. Vision Unit}

We use an OMRON D6T-44L device as a vision unit, which is composed of an array of 16 thermal sensors distributed in a $4 \times 4$ matrix, as shown in Fig. 3. This particular device has a horizontal viewing angle of $44.2^{\circ}$, and a vertical viewing angle of $45.7^{\circ}$.

The device is able to detect an object up to a distance of 10 meters. However, the detected temperatures vary as the distance increases. At a distance of $1 \mathrm{~cm}$, the measured surface temperature of the human body is approximately $32{ }^{\circ} \mathrm{C}$. On the other hand, at a distance of 5 meters, the measured temperature is of $28{ }^{\circ} \mathrm{C}$. For these reasons, the motion detection threshold and human temperature threshold should consider the distance between the sensor and the surface of the detected object. The D6T-44L device detects objects whose surface temperature is between $5{ }^{\circ} \mathrm{C}$ and $50{ }^{\circ} \mathrm{C}$, and withstands temperatures between $-10{ }^{\circ} \mathrm{C}$ and $60{ }^{\circ} \mathrm{C}$.

The communication interface that the sensor uses to communicate with other devices or control units is the InterIntegrated Circuit (I2C), which is a low speed communication bus developed by Phillips. This interface uses only two communication wires: the Serial Clock Line (SCL), and the Serial Data Line (SDA). The SCL is responsible for controlling data transmission through a clock signal, which determines the start 
and the end of a transmission. The $\mathrm{I} 2 \mathrm{C}$ channel works using the Master and Slave scheme. The master device is responsible over the SCL line. Up to 255 slave devices can be connected to the same bus, as long as there is a master device.

In the prototype, we used a personal (notebook) computer as the processing unit. The communication interface between the computer, and the I2C communication bus of the D6T$44 \mathrm{~L}$ device, was implemented using the Arduino platform. The Arduino programing language includes a library, named Wire, that simplifies the communication through the $\mathrm{I} 2 \mathrm{C}$ bus. One limitation of the Wire library is that it only supports a maximum of 32 bytes over the bus, whereas the D6T-44L device transmits 35 bytes for each read cycle. To solve this problem, a library that allows extending the capabilities of Wire was used, which is called WireExt.

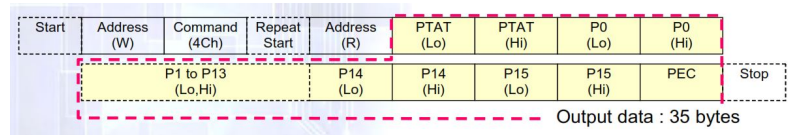

Figure 4. D6T-44L's output data structure

Fig. 4 shows the scheme under which the D6T-44L communicates. First, a byte containing the slave's device address is sent. Then, a byte that indicates the instructions is sent (this is done only once). Afterwards, it starts a cycle of data transmission, where the first two bytes are designated for the sensor's reference temperature, and two bytes of data for each element of the array. The last byte is known as PEC byte, which is used to check if the information was received correctly.

The data received by the sensor are converted to Celsius degrees and stored along with the reference temperature within an array of 17 elements. The data received by the processing unit are processed by the algorithms, which were developed using Processing V2. This is a programming language and an IDE based on he Java platform. The processing unit receives the data from the Arduino device via the serial port of the computer. The reading process goes on while there are still data available on the serial port.

\section{B. Experiments}

To perform the experiments to evaluate the proposed system, the following conditions were used in a controlled environment:

- The average ambient temperature was $25^{\circ} \mathrm{C}$.

- The floor surface temperature measured by the sensor varied from $24.1{ }^{\circ} \mathrm{C}$ to $24.5{ }^{\circ} \mathrm{C}$. That is, in normal conditions and without the presence of motion. The measured temperatures when there was no motion was in that range. If the collected temperature was outside this range, then we assumed that there was a moving object in the detection zone.

- The temperature of the human skin (wearing clothes) was in the range from $25.5^{\circ} \mathrm{C}$ to $28{ }^{\circ} \mathrm{C}$, when the sensor was place up to 4 meters away from the target. If the sensed temperature fall in that range, then the system assumed that the detected object was a pedestrian.
- The distance between the sensor and the ground was set to of 2.35 meters, which provided a sensing area of 1.2 square meters.

- With the purpose of the evaluation, the simplest scenario was considered: the detection of a single pedestrian moving in one direction. To detect more than one pedestrian, a multi-threaded application may be constructed, where each thread will be in charge of tracking the movements of one pedestrian.

- To evaluate the system, we assumed that the movement of the VRU always started in any of three points: the opposite side of the street, and both lateral sides. An object coming from the street was not considered of interest.

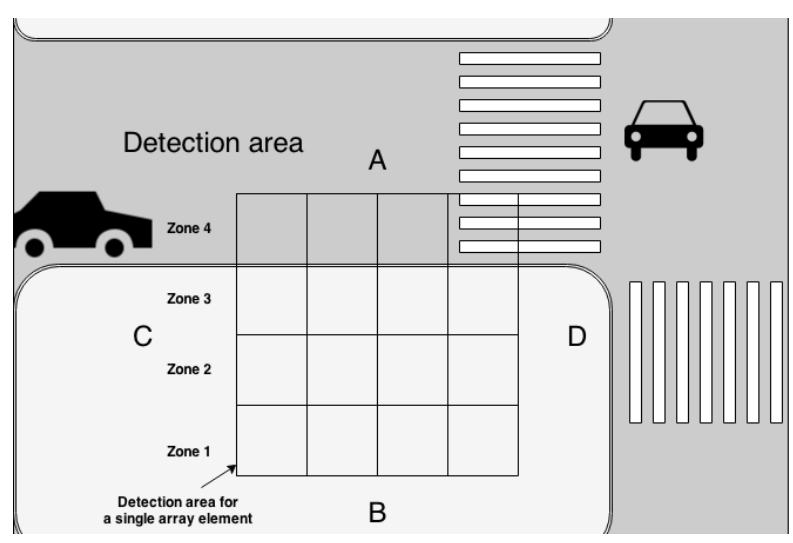

Figure 5. Detection Area

The motion detection threshold used in the experiments was set to $0.5{ }^{\circ} \mathrm{C}$. As mentioned before, the previous measurement and the current ones were compared. If the difference was greater than $0.5^{\circ} \mathrm{C}$, then the system concluded that a moving target was detected. To determine if the detected object is a pedestrian, we used the temperature range for human skin (from $25.5{ }^{\circ} \mathrm{C}$ to $30{ }^{\circ} \mathrm{C}$ ). To reduce the number of false positives, the range of human detection was modified to 26.5 ${ }^{\circ} \mathrm{C}$ to $30{ }^{\circ} \mathrm{C}$.

The detection area for a $4 \times 4$ array of sensors can be seen in Fig. 5. The point $\mathrm{A}$ is the closest to the street, whereas point $\mathrm{B}$ is the opposite side of the street. Points $\mathrm{C}$ and $\mathrm{D}$ are the lateral sides of detection area. The measurement for each element was done from left to right, and from bottom to top. The first row of the array was considered Zone 1, the next one was Zone 2, and so on. Zone 4 was assumed as the edge of the street.

The evaluation of the prototype was performed under the following scenarios (again refer to Fig. 5):

- Scenario 1: The pedestrian enters from B and walks straight to $\mathrm{A}$.

- Scenario 2: The pedestrian enters from B and stands still.

- Scenario 3: The pedestrian enters from B and is headed towards $\mathrm{C}$ or $\mathrm{D}$.

- Scenario 4: The pedestrian enters from B, remains motionless, and after a moment, he resumes his movement.

- Scenario 5: The pedestrian enters from C or D and goes towards A. 


\begin{tabular}{|c|c|c|c|c|c|}
\hline Scenario & Tests & Successful Tests & \% of Success & False Positive & False Negative \\
\hline \hline 1 & 25 & 20 & $80 \%$ & 5 & 0 \\
\hline 2 & 20 & 20 & $100 \%$ & 0 & 0 \\
\hline 3 & 40 & 40 & $100 \%$ & 0 & 0 \\
\hline 4 & 60 & 60 & $100 \%$ & 0 & 0 \\
\hline 5 & 40 & 40 & $100 \%$ & 0 & 0 \\
\hline 6 & 40 & 40 & $100 \%$ & 0 & 0 \\
\hline 7 & 40 & 40 & $100 \%$ & 0 & 10 \\
\hline 8 & 60 & 50 & $83 \%$ & 0 & 10 \\
\hline Total & 325 & 310 & $95 \%$ & 5 & \\
\hline
\end{tabular}

TABLE OF TESTS

- Scenario 6: The pedestrian enters from $\mathrm{C}$ or D and goes towards B.

- Scenario 7: The pedestrian enters from $\mathrm{C}$ or $\mathrm{D}$ and goes towards the opposite side.

- Scenario 8: The pedestrian enters from B, C or D and returns to the same side where he started.

To evaluate the performance of the system, we conducted 20 experiments for each scenario.

For Scenario 1, all tests were successful. We noted that in the cases where the VRU moves at high speed, (i.e., the pedestrian ran), the system sometimes failed to detect him. For Scenario 2, if a pedestrian enters to the detection zone from either side, the system waits for the VRU to start moving again, storing his status and last position. For these experiments, all the cases were detected successfully. For Scenario 3, which is similar to Scenario 1, also all tests were successfully detected. For this scenario, we conducted forty tests: twenty when the VRU was headed to $C$, and twenty when he was moving towards D. For Scenario 4, the position of the pedestrian when he stopped is stored, to allow the detection of the target when he begins to move again. In this set of experiments, twenty test where conducted when the user was headed towards A, twenty when he moved towards $\mathrm{C}$, and twenty when he directed towards D. Scenarios 5 and 6 are similar, and all their cases were successfully detected, as well as in the experiments for Scenario 7. However, for Scenario 8, the system failed in detecting $50 \%$ of the cases in which the VRU entered and returned from the same side. This can be explained by the fact that sometimes the VRU approached too much to the point at which it was considered that he had the intention to cross the street. In the case for which the VRU is coming and returning from $\mathrm{C}$ or D, all tests were successful. In Table I we can observe a summary of results obtained after conducting the experiments.

\section{CONCLUSiOnS AND Future WORK}

The rapid urban growth occurred in recent years has led to the emergence of new problems. For instance, road traffic injuries is the eighth leading cause of death globally, and the main cause of death for young people. The vulnerable road users (VRUs) group, which includes pedestrian and cyclists, has the higher risks of traffic accidents. Recently, the use of smart computing technologies have been proposed to build smart cities, as a strategy to mitigate the effects of the problems of the growing urban areas.
In this paper, we proposed a model to be used in smart cities, to detect if a VRU intends to cross a road at a risky zone, and to issue alerts to the vehicles nearby. The model is based on the use of Vehicular Ad hoc Networks (VANETs) technology, and on the use of non-contact temperature sensors. In order to evaluate the proposed system, we built a prototype using low cost and highly available components. The results of its showed that the model was able to detect a pedestrian at risk under controlled scenarios, and using low computational complexity algorithms.

As future work, we intend to build a prototype that will be able to detect more than one VRU moving in different directions, or moving at high speed.

\section{REFERENCES}

[1] United Nations Population Fund web site. http://www.unfpa.org.

[2] The Global status report on road safe. World Health Organization, 2013.

[3] Saif Al-Sultan, Moath M. Al-Doori, Ali H. Al-Bayatti, and Hussien Zedan. A comprehensive survey on vehicular ad hoc network. Journal of Network and Computer Applications, 37:380-392, 2014.

[4] O.M.J. Carsten. Can road transport informatics help vulnerable road users? In Proceedings of the IEEE Vehicle Navigation and Information Systems Conference, pages 1031-1036, Oct. 1991.

[5] Basel Fardi, Ulrich Neubert, Normen Giesecke, Holger Lietz, and Gerd Wanielik. A fusion concept of video and communication data for vru recognition. In Proceedings of the 11th IEEE International Conference on Information Fusion, pages 1-8, July 2008.

[6] K. Ch. Fuerstenberg, K. C. J. Dietmayer, and Willhoeft V. Pedestrian recognition in urban traffic using a vehicle based multilayer laser scanner. In Proceedings of the IEEE Intelligent Vehicle Symposium, pages 31-35, June 2002.

[7] Virginia Ruiz Garate, Roy Bours, and Kajetan Kietlinski. Numerical modeling of ada system for vulnerable road users protection based on radar and vision sensing. In Proceedings of the IEEE Intelligent Vehicles Symposium, pages 1150-1155, June 2012.

[8] Sarah Hasan and Edwin Yaz. Intelligent Transportation Systems. $\mathrm{PhD}$ thesis, University of Arkansas, 1999.

[9] Ronald Hughes, Herman Huang, Charles Zegeer, and Michael Cynecki. Evaluation of automated pedestrian detection at signalized intersections. Research Report, Department of Transportation, 2001.

[10] Daniel Meissner, Stephan Reuter, and Klaus Dietmayer. Real-time detection and tracking of pedestrians at intersections using a network of laserscanners. In Proceedings of the IEEE Intelligent Vehicles Symposium, pages 630-635, June 2012.

[11] D. J. Sherborne. Existing techniques for detecting vulnerable road users. Drive II Project V2005, 1992

[12] Christian Voigtmann, Sian Lun Lau, and Klaus David. Evaluation of a collaborative-based filter technique to proactively detect pedestrians at risk. In Proceedings of the IEEE Vehicular Technology Conference, pages 1-8, Sep 2012.

[13] Doug Washburn, Usman Sindhu, Stephanie Balaouras, Rachel A. Dines, Nick Hayes, and Lauren E. Nelson. Helping CIOs understand smart city initiatives: Defining the smart city, its drivers, and the role of the CIO. Forrester Research Inc., 2011 